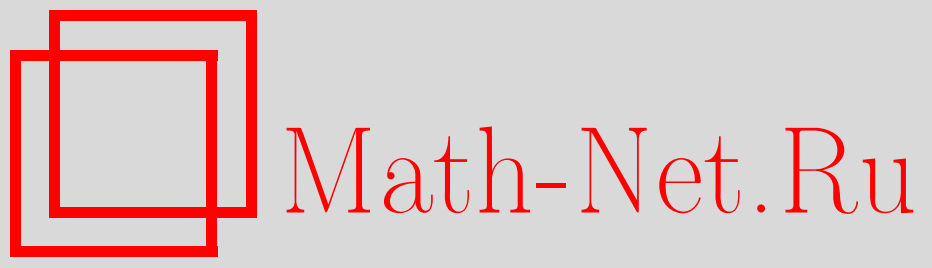

В. И. Афанасьев, О соотношении максимального и общего числа частиц в критическом ветвящемся процессе в случайной среде, Теория вероятн. и ее примен., 2003, том 48, выпуск 3, 435-452

DOI: https://doi.org/10.4213/tvp264

Использование Общероссийского математического портала MathNet.Ru подразумевает, что вы прочитали и согласны с пользовательским соглашением

http://www . mathnet.ru/rus/agreement

Параметры загрузки:

IP : 54.162.85.209

26 апреля 2023 г., 14:11:29

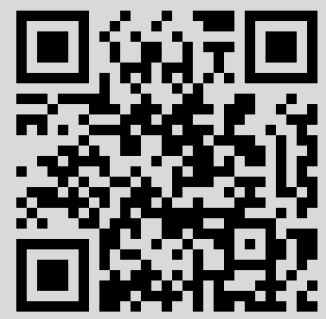


(C) $2003 \mathrm{r}$.

\author{
АФАНАСЬЕВ В. И.*
}

\title{
О СООТНОШЕНИИ МАКСИМАЛЬНОГО И ОБЩЕГО ЧИСЛА ЧАСТИЦ В КРИТИЧЕСКОМ ВЕТВЯЩЕМСЯ ПРОЦЕССЕ В СЛУЧАЙНОЙ СРЕДЕ ${ }^{1)}$
}

Пусть $\left\{\xi_{n}\right\}$ - критический ветвящийся процесс в случайной среде. Доказано, что при некоторых условиях отношение $\sum_{i=0}^{n} \xi_{i}$ к $\max _{0 \leqslant i \leqslant n} \xi_{i}$, рассматриваемое при условии $\left\{\xi_{n}>0\right\}$, сходится по распределению при $n \rightarrow \infty$ к некоторой случайной величине, принимающей значения из $(1,+\infty)$.

Ключевые слова $u$ фразы: ветвящийся процесс в случайной среде, условное случайное блуждание, условные предельные теоремы.

1. Пусть $\left\{\xi_{n}, n \in \mathbf{N}_{0}\right\}$ - ветвяшийся процесс в случайной среде $\left\{\pi_{n}, n \in \mathbf{N}_{0}\right\}$, где $\pi_{n}=\left\{\pi_{n}(0), \pi_{n}(1), \pi_{n}(2), \ldots\right\}, \pi_{n}(i) \geqslant 0, \sum_{i=0}^{\infty} \pi_{n}(i)=1$, $n \in \mathbf{N}_{0}=\{0,1,2, \ldots\}$, причем последовательности $\pi_{n}$ независимы и одинаково распределены при разных $n$. По определению это означает, что случайные величины $\xi_{n}$ являются неотрицательными целочисленными и

$$
\xi_{0}=1, \quad \mathbf{E}\left(s^{\xi_{n+1}} \mid \xi_{0}, \xi_{1}, \ldots, \xi_{n}, \pi_{0}, \pi_{1}, \ldots, \pi_{n}\right)=\left(f_{n}(s)\right)^{\xi_{n}},
$$

где $f_{n}(s)=\sum_{i=0}^{\infty} \pi_{n}(i) s^{i}, s \in[-1,1], n \in \mathbf{N}_{0}$. Положим

$$
X_{i}=\ln f_{i-1}^{\prime}(1), \quad \eta_{i}=\frac{f_{i-1}^{\prime \prime}(1)}{2 f_{i-1}^{\prime}(1)^{2}}, \quad i \in \mathbf{N} .
$$

Будем предполагать, что процесс $\left\{\xi_{n}\right\}$ является критическим, т.е. $\mathbf{E} X_{1}=0$, и выполнены условия: (a) конечны $\mathbf{E} X_{1}^{2}, \mathbf{E} \eta_{1}, \mathbf{E} X_{1} \eta_{1}$, причем $\mathbf{E} X_{1}^{2}=\sigma^{2}>0$; (b) $\mathbf{E} \exp \left(-X_{1}\right)<+\infty$.

Пусть $T=\inf \left\{n: \xi_{n}=0\right\}-$ момент вырождения $\left\{\xi_{n}\right\}$. Положим $m_{n}=\mathbf{E}_{\pi} \xi_{n}$ (символы $\mathbf{E}_{\pi}$ и $\mathbf{P}_{\pi}$ означают математическое ожидание и вероятность, вычисленные при условии, что среда $\left\{\pi_{n}\right\}$ фиксирована).

В работе автора [1] доказана следующая функциональная предельная теорема для критического ветвяшегося процесса в случайной среде.

* Московский энергетический институт, ул. Красноказарменная, 14, 105835 Москва, Россия; e-mail: viafan@mail.ru

1) Работа выполнена при поддержке РФФИ, код проекта 01-01-00649, и RFBR-DFG, код проекта 02-01-04002. 


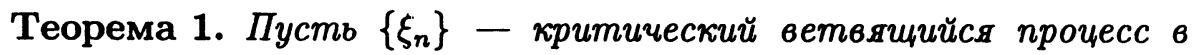
случайной среде и выполнены условия (a), (b), тогда при $n \rightarrow \infty$

$$
\left\{\frac{\xi_{[n t]}}{m_{[n t]}}, t \in[0,1] \mid T>n\right\} \stackrel{\mathscr{D}}{\longrightarrow} \mu,
$$

где $\mu$ - случайный прочесс с неотричательныции постоянными реализачиями на $(0,1]$, причем $\mathbf{P}\{\mu>0\}>0$; сходимость в (1) надо понимать как сходимость по распределению в пространстве $D[u, 1]$ с топологией Скорохода при любом фиксированном $u \in(0,1)$.

В частном случае, когда производящие функции $f_{n}(s), n \in \mathbf{N}_{0}$, являются дробно-линейными, в работе автора [2] показано, что $\mu>0$ п.н.

Из теоремы 1 вытекает следующая функциональная предельная теорема для логарифма критического ветвящегося процесса в случайной среде.

Теорема 2. Пусть $\left\{\xi_{n}\right\}-$ - критический ветвяиийся прочесс в случайной среде, выполнены условия (a), (b) $u \mu>0$ п.н. Тогда при $n \rightarrow \infty$

$$
\left\{\frac{\ln \xi_{[n t]}}{\sigma \sqrt{n}}, t \in[0,1] \mid T>n\right\} \stackrel{\mathscr{D}}{\longrightarrow}\left\{W^{+}(t), t \in[0,1]\right\}
$$

где $\left\{W^{+}(t)\right\}$ - броуновская извилина; сходимость в (2) надо понимать как сходимость по распределению в пространстве $D[0,1]$ с топологией Cкорохода.

Д ок а з а т ел ь с т в о. В работе [2] приведен вывод соотношения (2) из соотношения (1) для случая, когда производящие функции $f_{n}(s), n \in \mathbf{N}_{0}$, дробно-линейны. Точно так же этот вывод осуществляется и в условиях теоремы 2. При этом надо использовать, вопервых, полученную Керстингом и Гейгером [3] асимптотическую формулу: при выполнении условия (а) и $n \rightarrow \infty$

$$
\mathbf{P}\{T>n\} \sim \frac{c_{2}}{\sqrt{n}}
$$

где $c_{2}$ - положительная постоянная; и, во-вторых, следующие леммы 1 и 2, обобщающие соответственно леммы 6 и 7 из [2].

Чтобы сформулировать эти леммы, введем так называемое сопровождаюшее случайное блуждание $S_{0}=0, S_{n}=X_{1}+\cdots+X_{n}, n \in \mathrm{N}$. Оно имеет нулевой снос, поскольку процесс $\left\{\xi_{n}\right\}$ является критическим. Пусть $\tau_{0}=0, \tau_{1}, \tau_{2}, \ldots$ - строгие нижние лестничные моменты этого блуждания, т.е.

$$
\tau_{1}=\inf \left\{n: S_{n}<0\right\}, \quad \tau_{2}=\inf \left\{n>\tau_{1}: S_{n}<S_{\tau_{1}}\right\}, \ldots
$$

В работе М.В. Козлова [4] установлено, что если $\mathbf{E} X_{1}=0,0<\mathbf{E} X_{1}^{2}<$ $+\infty$, то при любом $j \in \mathbf{N}_{0}$ и $n \rightarrow \infty$

$$
\mathbf{P}\left\{\tau_{j} \leqslant n<\tau_{j+1}\right\} \sim \frac{c_{1}}{\sqrt{n}}
$$


где $c_{1}$ - положительная постоянная. Следующее утверждение доказано в $[1$, лемма 10$]$.

Лемма 1. Пусть $\left\{\xi_{n}\right\}$ - критический ветвящийся прочесс в случайной среде и выполнено условие (а). Если $\left\{A_{n}\right\}-$ такая последовательность случайных событий, что при яюбом $j \in \mathbf{N}_{0}$ существует $\lim _{n \rightarrow \infty} \mathbf{P}\left\{A_{n}, T>n \mid \tau_{j} \leqslant n<\tau_{j+1}\right\}=l_{j}$, то существует

$$
\lim _{n \rightarrow \infty} \mathbf{P}\left\{A_{n} \mid T>n\right\}=\frac{c_{1}}{c_{2}} \sum_{j=0}^{\infty} l_{j}
$$

где $c_{1}, c_{2}$ - постоянные из соотношений (4) $u$ (3) соответственно.

Положим

$$
Y_{n}(t)=\frac{S_{[n t]}}{\sigma \sqrt{n}}, \quad t \in[0,+\infty), \quad n \in \mathbf{N} .
$$

Лемма 2. Пусть $\left\{\xi_{n}\right\}$ - критический ветвящийся прочесс в случайной среде и выполнено условие (a), тогда при $n \rightarrow \infty$

$$
\left\{Y_{n}(t), t \in[0,+\infty) \mid T>n\right\} \stackrel{\mathscr{D}}{\longrightarrow}\left\{W^{+}(t), t \in[0,+\infty)\right\}
$$

причем сходимость в (5) надо понимать как сходимость по распределению в пространстве $D[0,+\infty)$ с топологией Cкорохода.

Сходимость конечномерных распределений в (5) установлена В. А. Ватутиным [5, лемма 5]. Плотность соответствующих вероятностных мер доказывается так же, как в [2, лемма 7].

3 а м е ч а н и е 1. Другой вариант условий, при которых выполняется соотношение (2), приведен в работе М. В. Козлова [6]. Там предполагается, что выполнены условия (a), (b) и $\mathbf{E} X_{1}^{4}<+\infty$.

3 а м е ч а н и е 2 . Из соотношений (2), (3) вытекают многочисленные следствия, установленные автором в работах [7]-[9]. В силу вышесказанного используемое в этих работах предположение о том, что производящие функции $f_{n}(s), n \in \mathbf{N}_{0}$, дробно-линейны, оказывается излишним.

Цель настоящей работы - изучение соотношения максимального числа частиц $\max _{0 \leqslant i \leqslant n} \xi_{i}$ и общего числа частиц $\sum_{i=0}^{n} \xi_{i}$ в критическом ветвящемся процессе в случайной среде $\left\{\xi_{n}\right\}$, рассматриваемом при условии $\{T>n\}$. Оказывается, с ростом числа поколений $n$ роль максимального поколения не уменьшается и доля числа частиц из максимального поколения в общем числе частиц стабилизируется и является положительной.

Более точно, имеет место следующий результат.

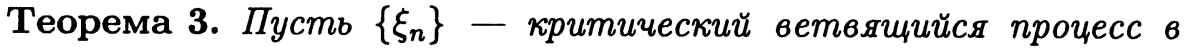
случайной среде, выполнены условия (a), (b) $u \mu>0$ п.н., тогда 
$n p u n \rightarrow \infty$

$$
\left\{\frac{\sum_{i=0}^{n} \xi_{i}}{\max _{0 \leqslant i \leqslant n} \xi_{i}} \mid T>n\right\} \stackrel{\mathscr{D}}{\longrightarrow} \varphi,
$$

где $\varphi$ - случайная величина, принимающая значения из $(1,+\infty)$, причем $\varphi$ есть сумма двух независимых случайных величин $\hat{\varphi}$ и $\check{\varphi}$ с функчиями распределения соответственно

$$
\begin{aligned}
& \widehat{F}(x)=\lim _{n \rightarrow \infty} \mathbf{P}\left\{\frac{1}{2}+\sum_{i=1}^{n} e^{-S_{i}} \leqslant x \mid S_{1}>0, \ldots, S_{n}>0\right\}, \\
& \check{F}(x)=\lim _{n \rightarrow \infty} \mathbf{P}\left\{\frac{1}{2}+\sum_{i=1}^{n} e^{S_{i}} \leqslant x \mid S_{1} \leqslant 0, \ldots, S_{n} \leqslant 0\right\} .
\end{aligned}
$$

3 а м е ч а н и е 3. Цоказательство того, что пределы (7) и (8) существуют и задают собственные распределения на $\left(\frac{1}{2},+\infty\right)$, можно найти в работе автора [10].

3 а м е ч а н и е 4 . Теорема 3 доставляет пока единственный пример результата, который доказывается с применением теоремы 1 , но не может быть выведен из теоремы 2.

2. Доказательству теоремы 3 предпошлем ряд лемм. Следуюший результат, доказанный в [1, лемма 12], существенно дополняет теорему 1 и играет важную роль в дальнейшем.

Лемма 3. Если $\left\{\xi_{n}\right\}-$ - критический ветвящийся прочесс в случайной среде и выполнень условия (a), (b), то при любом фиксированном $\delta \in(0,1)$ u $n \rightarrow \infty$

$$
\left\{\sup _{\delta \leqslant t \leqslant 1}\left|\frac{\xi_{[n t]}}{m_{[n t]}}-\frac{\xi_{n}}{m_{n}}\right| \mid T>n\right\} \stackrel{\mathscr{D}}{\longrightarrow} 0 .
$$

Лемма 4. В условиях леммы 3 при $n \rightarrow \infty$

$$
\left\{\frac{\sum_{i=0}^{n} \xi_{i}}{\sum_{i=0}^{n} m_{i}}-\frac{\xi_{n}}{m_{n}} \mid T>n\right\} \stackrel{\mathscr{D}}{\longrightarrow} 0 .
$$

Доказате льст в о. В силу леммы 3 при фиксированных $\delta \in(0,1)$ и $\varepsilon \in(0,+\infty)$

$$
\lim _{n \rightarrow \infty} \mathbf{P}\left\{\left|\frac{\xi_{i}}{m_{i}}-\frac{\xi_{n}}{m_{n}}\right| \leqslant \varepsilon, \forall i \in(\delta n, n] \mid T>n\right\}=1 .
$$

Положим $a_{n}=\xi_{n} / m_{n}$. Если $\left|\xi_{i} / m_{i}-\xi_{n} / m_{n}\right| \leqslant \varepsilon$ при любом $i \in(\delta n, n]$, то $a_{n}-\varepsilon \leqslant \xi_{i} / m_{i} \leqslant a_{n}+\varepsilon$ при любом $i \in(\delta n, n]$, и поэтому

$$
\begin{aligned}
a_{n}-\varepsilon & =\frac{\sum_{i==[\delta n]+1}^{n}\left(a_{n}-\varepsilon\right) m_{i}}{\sum_{i=[\delta n]+1}^{n} m_{i}} \leqslant \frac{\sum_{i=[\delta n]+1}^{n} \xi_{i}}{\sum_{i=[\delta n]+1}^{n} m_{i}} \\
& \leqslant \frac{\sum_{i=[\delta n]+1}^{n}\left(a_{n}+\varepsilon\right) m_{i}}{\sum_{i=[\delta n]+1}^{n} m_{i}}=a_{n}+\varepsilon .
\end{aligned}
$$


Из соотношений (9) и (10) следует, что при фиксированных $\delta \in(0,1)$ и $\varepsilon \in(0,+\infty)$

$$
\lim _{n \rightarrow \infty} \mathbf{P}\left\{\left|\frac{\sum_{i=[\delta n]+1}^{n} \xi_{i}}{\sum_{i=[\delta n]+1}^{n} m_{i}}-a_{n}\right| \leqslant \varepsilon \mid T>n\right\}=1 .
$$

Далее, поскольку при фиксированном $t \in[0,1]$ справедливы неравенства $\max _{0 \leqslant i \leqslant t n} \xi_{i} \leqslant \sum_{i=0}^{[t n]} \xi_{i} \leqslant(1+t n) \max _{0 \leqslant i \leqslant t n} \xi_{i}$, то ввиду теоремы 2 при фиксированном $\delta \in(0,1)$ и $n \rightarrow \infty$

$$
\begin{gathered}
\left\{\frac{\ln \sum_{i=0}^{[\delta n]} \xi_{i}}{\sigma \sqrt{n}}, \frac{\ln \sum_{i=[\delta n]+1}^{n} \xi_{i}}{\sigma \sqrt{n}} \mid T>n\right\} \\
\stackrel{\mathscr{D}}{\longrightarrow}\left\{\max _{0 \leqslant t \leqslant \delta} W^{+}(t), \max _{\delta \leqslant t \leqslant 1} W^{+}(t)\right\} .
\end{gathered}
$$

Следовательно, при $n \rightarrow \infty$

$$
\left\{\frac{\ln \sum_{i=0}^{[\delta n]} \xi_{i}}{\ln \sum_{i=[\delta n]+1}^{n} \xi_{i}} \mid T>n\right\} \stackrel{\mathscr{D}}{\longrightarrow} \frac{\max _{0 \leqslant t \leqslant \delta} W^{+}(t)}{\max _{\delta \leqslant t \leqslant 1} W^{+}(t)} .
$$

Поскольку случайная величина в правой части сходится п.н. к нулю при $\delta \rightarrow 0$, то при фиксированном $\varepsilon_{0} \in(0,+\infty)$

$$
\lim _{\delta \rightarrow 0} \lim _{n \rightarrow \infty} \mathbf{P}\left\{\frac{\ln \sum_{i=0}^{[\delta n]} \xi_{i}}{\ln \sum_{i=[\delta n]+1}^{n} \xi_{i}}>\varepsilon_{0} \mid T>n\right\}=0 .
$$

Очевидно, что

$$
\frac{\ln \sum_{i=0}^{[\delta n]} \xi_{i}}{\ln \sum_{i=[\delta n]+1}^{n} \xi_{i}}>\varepsilon_{0} \Longleftrightarrow \frac{\sum_{i=0}^{[\delta n]} \xi_{i}}{\sum_{i=[\delta n]+1}^{n} \xi_{i}}>\frac{1}{\left(\sum_{i=[\delta n]+1}^{n} \xi_{i}\right)^{1-\varepsilon_{0}}} .
$$

В силу соотношения (12) при фиксированных $\delta, \varepsilon_{0} \in(0,1)$ и $n \rightarrow \infty$

$$
\left\{\frac{1}{\left(\sum_{i=[\delta n]+1}^{n} \xi_{i}\right)^{1-\varepsilon_{0}}} \mid T>n\right\} \stackrel{\mathscr{D}}{\longrightarrow} 0 .
$$

Из соотношений (13)-(15) находим, что при фиксированном $\varepsilon \in(0,+\infty)$

$$
\varlimsup_{\delta \rightarrow 0} \varlimsup_{n \rightarrow \infty} \mathbf{P}\left\{\frac{\sum_{i=0}^{[\delta n]} \xi_{i}}{\sum_{i=[\delta n]+1}^{n} \xi_{i}}>\varepsilon \mid T>n\right\}=0 .
$$

Заметим что $m_{i}=\exp S_{i}, i \in \mathbf{N}_{0}$, поэтому аналогично соотношению (16) получаем из леммы 2 , что при фиксированном $\varepsilon \in(0,+\infty)$

$$
\varlimsup_{\delta \rightarrow 0} \varlimsup_{n \rightarrow \infty} \mathbf{P}\left\{\frac{\sum_{i=0}^{[\delta n]} m_{i}}{\sum_{i=[\delta n]+1}^{n} m_{i}}>\varepsilon \mid T>n\right\}=0 .
$$


Из представления

$$
\begin{aligned}
\frac{\sum_{i=0}^{n} \xi_{i}}{\sum_{i=0}^{n} m_{i}} & =\frac{\sum_{i=0}^{[\delta n]} \xi_{i}+\sum_{i=[\delta n]+1}^{n} \xi_{i}}{\sum_{i=0}^{[\delta]} m_{i}+\sum_{i=[\delta n]+1}^{n} m_{i}} \\
& =\frac{\sum_{i=[\delta n]+1}^{n} \xi_{i}}{\sum_{i=[\delta n]+1}^{n} m_{i}} \frac{1+\sum_{i=1}^{[\delta n]} \xi_{i} / \sum_{i=[\delta n]+1}^{n} \xi_{i}}{1+\sum_{i=1}^{[\delta n]} m_{i} / \sum_{i=[\delta n]+1}^{n} m_{i}}
\end{aligned}
$$

и соотношений $(11),(16),(17)$ следует утверждение леммы 4.

Лемма 5. В условиях леммы 3 при $n \rightarrow \infty$

$$
\left\{\frac{\max _{0 \leqslant i \leqslant n} \xi_{i}}{\max _{0 \leqslant i \leqslant n} m_{i}}-\frac{\xi_{n}}{m_{n}} \mid T>n\right\} \stackrel{\mathscr{D}}{\longrightarrow} 0 .
$$

Доказ ательство. В силу леммы 3 при фиксированных $\delta \in(0,1)$ и $\varepsilon \in(0,+\infty)$

$$
\lim _{n \rightarrow \infty} \mathbf{P}\left\{\left|\xi_{i} m_{i}^{-1}-a_{n}\right|<\varepsilon \text { при любом } i \in(\delta n, n] \mid T>n\right\}=1,
$$

где $a_{n}=\xi_{n} / m_{n}$. Если $\left|\xi_{i} / m_{i}-a_{n}\right| \leqslant \varepsilon$ при любом $i \in(\delta n, n]$ и $\max _{\delta n<i \leqslant n} \xi_{i}$ достигается при $i=i_{0}$, то $\xi_{i} \leqslant \xi_{i_{0}}, i \in(\delta n, n]$;

$\left(a_{n}-\varepsilon\right) m_{i_{0}} \leqslant \xi_{i_{0}} \leqslant\left(a_{n}+\varepsilon\right) m_{i_{0}} ; \quad\left(a_{n}-\varepsilon\right) m_{i} \leqslant \xi_{i} \leqslant\left(a_{n}+\varepsilon\right) m_{i}, \quad i \in(\delta n, n]$.

Поэтому при $i \in(\delta n, n]$

и, следовательно,

$$
m_{i_{0}} \geqslant \frac{\xi_{i_{0}}}{a_{n}+\varepsilon} \geqslant \frac{\xi_{i}}{a_{n}+\varepsilon} \geqslant \frac{\left(a_{n}-\varepsilon\right) m_{i}}{a_{n}+\varepsilon}
$$

$$
m_{i_{0}} \geqslant \frac{a_{n}-\varepsilon}{a_{n}+\varepsilon} \max _{\delta n<i \leqslant n} m_{i} .
$$

С другой стороны, $m_{i_{0}} \leqslant \max _{\delta n<i \leqslant n} m_{i}$. Таким образом,

$$
\frac{\left(a_{n}-\varepsilon\right)^{2}}{a_{n}+\varepsilon} \leqslant \frac{a_{n}-\varepsilon}{a_{n}+\varepsilon} \frac{\xi_{i_{0}}}{m_{i_{0}}} \leqslant \frac{\xi_{i_{0}}}{\max _{\delta n<i \leqslant n} m_{i}} \leqslant \frac{\xi_{i_{0}}}{m_{i_{0}}} \leqslant a_{n}+\varepsilon,
$$

но это означает, что $\left(a_{n}-\varepsilon\right)^{2} /\left(a_{n}+\varepsilon\right) \leqslant \max _{\delta n<i \leqslant n} \xi_{i} / \max _{\delta n<i \leqslant n} m_{i} \leqslant$ $a_{n}+\varepsilon$. Откуда, учитывая, что

$$
\frac{\left(a_{n}-\varepsilon\right)^{2}}{a_{n}+\varepsilon}-a_{n}=\frac{\varepsilon^{2}-3 \varepsilon a_{n}}{a_{n}+\varepsilon} \geqslant-3 \varepsilon,
$$

получаем, что

$$
a_{n}-3 \varepsilon \leqslant \frac{\max _{\delta n<i \leqslant n} \xi_{i}}{\max _{\delta n<i \leqslant n} m_{i}} \leqslant a_{n}+3 \varepsilon .
$$

Из соотношений (18), (19) следует, что при фиксированных $\delta \in(0,1)$ и $\varepsilon \in(0,+\infty)$

$$
\lim _{n \rightarrow \infty} \mathbf{P}\left\{\left|\frac{\max _{\delta n<i \leqslant n} \xi_{i}}{\max _{\delta n<i \leqslant n} m_{i}}-a_{n}\right| \leqslant 3 \varepsilon \mid T>n\right\}=1
$$


Далее, ввиду теоремы 2 при фиксированном $\delta \in(0,1)$ и $n \rightarrow \infty$

$$
\begin{aligned}
& \left\{\frac{\ln \max _{0 \leqslant i \leqslant \delta n} \xi_{i}}{\sigma \sqrt{n}}, \frac{\ln \max _{\delta n<i \leqslant n} \xi_{i}}{\sigma \sqrt{n}} \mid T>n\right\} \\
& \stackrel{\mathscr{D}}{\longrightarrow}\left\{\max _{0 \leqslant t \leqslant \delta} W^{+}(t), \max _{\delta \leqslant t \leqslant 1} W^{+}(t)\right\} .
\end{aligned}
$$

Откуда вытекает, что при $n \rightarrow \infty$

$$
\lim _{n \rightarrow \infty} \mathbf{P}\left\{\max _{0 \leqslant i \leqslant \delta n} \xi_{i}<\max _{\delta n<i \leqslant n} \xi_{i} \mid T>n\right\}=\mathbf{P}\left\{\max _{0 \leqslant t \leqslant \delta} W^{+}(t)<\max _{\delta \leqslant t \leqslant 1} W^{+}(t)\right\} .
$$

Поскольку вероятность в правой части стремится к 1 при $\delta \rightarrow 0$, получаем, что

$$
\lim _{\delta \rightarrow 0} \lim _{n \rightarrow \infty} \mathbf{P}\left\{\max _{0 \leqslant i \leqslant \delta n} \xi_{i}<\max _{\delta n<i \leqslant n} \xi_{i} \mid T>n\right\}=1 .
$$

Аналогично из леммы 2 находим, что

$$
\lim _{\delta \rightarrow 0} \lim _{n \rightarrow \infty} \mathbf{P}\left\{\max _{0 \leqslant i \leqslant \delta n} m_{i}<\max _{\delta n<i \leqslant n} m_{i} \mid T>n\right\}=1 .
$$

Из соотношений (20)-(22) следует утверждение леммы 5 .

Положим при $\delta \in[0,1)$ и $n \in \mathbf{N}_{0}$

$$
\varphi_{n}(\delta)=\frac{\sum_{i=[\delta n]}^{n} e^{S_{i}}}{\max _{[\delta n] \leqslant i \leqslant n} e^{S_{i}}}
$$

и вместо $\varphi_{n}(0)$ будем писать $\varphi_{n}$.

Лемма 6. Если $\mathbf{E} X_{1}=0,0<\mathbf{E} X_{1}^{2}<+\infty$, то при любом $x \in$ $(0,+\infty)$ и любом $\varphi$-непрерывном борелевском множестве $B \subset \mathbf{R}$

$$
\lim _{n \rightarrow \infty} \mathbf{P}\left\{\varphi_{n} \in B \mid \min _{0 \leqslant i \leqslant n} \frac{S_{i}}{\sigma \sqrt{n}} \geqslant-x\right\}=\mathbf{P}\{\varphi \in B\},
$$

где $\varphi-$ - жа же слиайная величина, что и в соотношении (6); сходимость в (23) равномерна по $x$ на любом отрезке $[a, b] \subset(0,+\infty)$.

Д о к а з а т е л ь с т в о. Пусть $T_{M}$ - момент первого достижения максимума для случайной последовательности $S_{0}, S_{1}, \ldots, S_{n}$, т.е.

$$
T_{M}=\inf \left\{i: S_{i}=\max _{0 \leqslant k \leqslant n} S_{k}\right\} \text {. }
$$

Положим $\widehat{S}_{i}=S_{T_{M}}-S_{T_{M-i}}, \check{S}_{i}=S_{T_{M}+i}-S_{T_{M}}, i=0,1,2, \ldots$ Очевидно, что $\varphi_{n}=\widehat{\varphi}_{n}+\check{\varphi}_{n}$, где $\widehat{\varphi}_{n}=\frac{1}{2}+\sum_{i=1}^{T_{M}} e^{-\widehat{S}_{i}}, \check{\varphi}_{n}=\frac{1}{2}+\sum_{i=1}^{n-T_{M}} e^{\check{S}_{i}}$. Рассмотрим совместное распределение случайных величин $\widehat{\varphi}_{n}$ и $\check{\varphi}_{n}$ при условии $\left\{\min _{0 \leqslant i \leqslant n} S_{i} /(\sigma \sqrt{n}) \geqslant-x\right\}$. Пусть $B_{1}-\hat{\varphi}$-непрерывное, а $B_{2}-$ 
$\check{\varphi}$-непрерывное борелевские множества на прямой (по поводу $\hat{\varphi}, \check{\varphi}$ см. формулировку теоремы 3 ). Покажем, что

$\lim _{n \rightarrow \infty} \mathbf{P}\left\{\widehat{\varphi}_{n} \in B_{1}, \check{\varphi}_{n} \in B_{2} \mid \min _{0 \leqslant i \leqslant n} \frac{S_{i}}{\sigma \sqrt{n}} \geqslant-x\right\}=\mathbf{P}\left\{\hat{\varphi} \in B_{1}\right\} \mathbf{P}\left\{\check{\varphi} \in B_{2}\right\}$,

откуда будет следовать утверждение доказываемой леммы.

При условии $\left\{\min _{0 \leqslant i \leqslant n} S_{i} /(\sigma \sqrt{n}) \geqslant-x\right\}$ и достаточно больших $n$ с вероятностью, близкой к единице, $T_{M} / n \in(\alpha, 1-\alpha], S_{T_{M}} /(\sigma \sqrt{n}) \in(\beta, 1 / \beta]$, где $\alpha$ и $\beta$ положительны и достаточно малы. Это следует из принципа инвариантности Донскера-Прохорова: в условиях доказываемой леммы при $n \rightarrow \infty$

$$
\left\{\frac{S_{[n t]}}{\sigma \sqrt{n}}, t \in[0,+\infty)\right\} \stackrel{\mathscr{D}}{\longrightarrow}\{W(t), t \in[0,+\infty)\},
$$

где $\{W(t)\}$ - стандартное броуновское движение, выходящее из нуля. Поэтому вместо $\mathbf{P}\left\{\hat{\varphi}_{n} \in B_{1}, \check{\varphi}_{n} \in B_{2} \mid \min _{0 \leqslant i \leqslant n} S_{i} /(\sigma \sqrt{n}) \geqslant-x\right\}$ рассмотрим близкую к ней вероятность

$$
\begin{aligned}
\mathbf{P}\left\{\widehat{\varphi}_{n} \in B_{1}, \check{\varphi}_{n} \in B_{2}, \frac{T_{M}}{n} \in(\alpha, 1-\alpha], \frac{S_{T_{M}}}{\sigma \sqrt{n}} \in\left(\beta, \frac{1}{\beta}\right] \mid \min _{0 \leqslant i \leqslant n} \frac{S_{i}}{\sigma \sqrt{n}} \geqslant-x\right\} \\
=\frac{P_{n}(\alpha, \beta)}{\mathbf{P}\left\{\min _{0 \leqslant i \leqslant n} S_{i} /(\sigma \sqrt{n}) \geqslant-x\right\}},
\end{aligned}
$$

где $P_{n}(\alpha, \beta)=\mathbf{P}\left\{\hat{\varphi}_{n} \in B_{1}, \check{\varphi}_{n} \in B_{2}, T_{M} / n \in(\alpha, 1-\alpha], S_{T_{M}} /(\sigma \sqrt{n}) \in\right.$ $\left.(\beta, 1 / \beta], \min _{0 \leqslant i \leqslant n} S_{i} /(\sigma \sqrt{n}) \geqslant-x\right\}$. Очевидно, что

$$
\begin{aligned}
P_{n}(\alpha, \beta)=\sum_{k=[\alpha n]+1}^{[(1-\alpha) n]} \mathbf{P}\left\{\widehat{\varphi}_{n} \in B_{1}, \check{\varphi}_{n} \in B_{2}, T_{M}=k,\right. \\
\left.\frac{S_{k}}{\sigma \sqrt{n}} \in\left(\beta, \frac{1}{\beta}\right], \min _{0 \leqslant i \leqslant n} \frac{S_{i}}{\sigma \sqrt{n}} \geqslant-x\right\} .
\end{aligned}
$$

Разобьем промежуток $(\alpha, 1-\alpha]$ точками $t_{0}=\alpha<t_{1}<t_{2}<\cdots<t_{l}=$ $1-\alpha$, а промежуток $(\beta, 1 / \beta]$ точками $y_{0}=\beta<y_{1}<y_{2}<\cdots<y_{m}=1 / \beta$. Положим

$$
\begin{array}{r}
Q_{n}(k, j)=\mathbf{P}\left\{\widehat{\varphi}_{n} \in B_{1}, \check{\varphi}_{n} \in B_{2}, T_{M}=k,\right. \\
\left.\frac{S_{k}}{\sigma \sqrt{n}} \in\left(y_{j}, y_{j+1}\right], \min _{0 \leqslant i \leqslant n} \frac{S_{i}}{\sigma \sqrt{n}} \geqslant-x\right\} .
\end{array}
$$

Тогда

$$
P_{n}(\alpha, \beta)=\sum_{k=[\alpha n]+1}^{[(1-\alpha) n]} \sum_{j=0}^{m-1} Q_{n}(k, j)
$$


Разбивая траекторию случайного блуждания $\left\{S_{i}\right\}$ на участки до момента $T_{M}$ и после него, получаем, что

$$
\begin{aligned}
& Q_{n}(k, j) \leqslant \mathbf{P}\left\{\frac{1}{2}+\sum_{i=0}^{k} e^{-\widehat{S}_{i}}\right. \in B_{1}, \frac{\widehat{S}_{k}}{\sigma \sqrt{n}} \in\left(y_{j}, y_{j+1}\right], \widehat{\tau}_{1}>k, \\
&\left.\max _{0 \leqslant i \leqslant k} \frac{\widehat{S}_{i}}{\sigma \sqrt{n}} \leqslant \frac{\widehat{S}_{k}}{\sigma \sqrt{n}}+x\right\} \mathbf{P}\left\{\frac{1}{2}+\sum_{i=1}^{n-k} e^{\check{S}_{i}} \in B_{2}, \check{\tau}_{1}>n-k,\right. \\
&\left.\min _{0 \leqslant i \leqslant n-k} \frac{\check{S}_{i}}{\sigma \sqrt{n}} \geqslant-\left(y_{j+1}+x\right)\right\},
\end{aligned}
$$

где в правой части на случайные последовательности $\left\{\widehat{S}_{i}\right\}$ и $\left\{\check{S}_{i}\right\}$ можно смотреть как на две точные вероятностные копии случайного блуждания $\left\{S_{i}\right\}$ и $\widehat{\tau}_{1}=\inf \left\{i>0: \widehat{S}_{i} \leqslant 0\right\}, \check{\tau}_{2}=\inf \left\{i>0: \check{S}_{i}>0\right\}$.

В работе автора [10] показано, что при условии $\left\{S_{1}>0, \ldots, S_{n}>0\right\}$ случайная величина $\frac{1}{2}+\sum_{i=0}^{n} \exp \left(-S_{i}\right)$ и случайный процесс $\left\{S_{[n t]} /(\sigma \sqrt{n})\right.$, $t \in[0,+\infty)\}$ при $n \rightarrow \infty$ асимптотически независимы (в смысле конечномерных распределений). Аналогичный результат справедлив для случайной величины $\frac{1}{2}+\sum_{i=0}^{n} \exp S_{i}$ и случайного процесса $\left\{-S_{[n t]} /(\sigma \sqrt{n})\right.$, $t \in[0,+\infty)\}$, рассматриваемых при условии $\left\{S_{1} \leqslant 0, \ldots, S_{n} \leqslant 0\right\}$. В [10] также показано, что случайные величины

$$
\left\{\frac{1}{2}+\sum_{i=0}^{n} e^{-S_{i}} \mid S_{1}>0, \ldots, S_{n}>0\right\} \quad \text { и }\left\{\frac{1}{2}+\sum_{i=0}^{n} e^{S_{i}} \mid S_{1} \leqslant 0, \ldots, S_{n} \leqslant 0\right\}
$$

сходятся по распределению при $n \rightarrow \infty$ (к случайным величинам $\widehat{\varphi}$ и $\check{\varphi}$ соответственно). Далее, в условиях доказываемой леммы справедлив условный принцип инвариантности Иглхарта (см. [11]): при $n \rightarrow \infty$

$$
\left\{\frac{S_{[n t]}}{\sigma \sqrt{n}}, t \in[0,+\infty) \mid S_{1}>0, \ldots, S_{n}>0\right\} \stackrel{\mathscr{D}}{\longrightarrow}\left\{W^{+}(t), t \in[0,+\infty)\right\} .
$$

Аналогичный результат справедлив для процесса $\left\{-S_{[n t]} /(\sigma \sqrt{n}), t \in\right.$ $[0,+\infty)\}$ при условии $\left\{S_{1} \leqslant 0, \ldots, S_{n} \leqslant 0\right\}$. Наконец (см. (4) при $j=0$ ), при $n \rightarrow \infty$

$$
\mathbf{P}\left\{\widehat{\tau}_{1}>n\right\} \sim \frac{\widehat{c}}{\sqrt{n}}, \quad \mathbf{P}\left\{\check{\tau}_{1}>n\right\} \sim \frac{\check{c}}{\sqrt{n}} .
$$

Зафиксируем $\varepsilon>0$, тогда в силу сказанного существует такое натуральное число $N(\varepsilon)$, что при всех $n \geqslant N(\varepsilon)$

$$
\begin{aligned}
Q_{n}(k, j) \leqslant & (1+\varepsilon) \mathbf{P}\left\{\hat{\varphi} \in B_{1}\right\} \mathbf{P}\left\{\check{\varphi} \in B_{2}\right\} \frac{\widehat{c}}{\sqrt{n t_{r}}} \frac{\check{c}}{\sqrt{n\left(1-t_{r+1}\right)}} \\
& \times \mathbf{P}\left\{W^{+}(1) \in\left(\frac{y_{j}}{\sqrt{t_{r+1}}}, \frac{y_{j+1}}{\sqrt{t_{r}}}\right], \sup _{0 \leqslant t \leqslant 1}\left[W^{+}(t)-W^{+}(1)\right] \leqslant \frac{x}{\sqrt{t_{r}}}\right\} \\
& \times \mathbf{P}\left\{\sup _{0 \leqslant t \leqslant 1} W^{+}(t) \geqslant \frac{y_{j+1}+x}{\sqrt{1-t_{r+1}}}\right\},
\end{aligned}
$$


если $k \in\left(t_{r} n, t_{r+1} n\right], r=0,1, \ldots, l-1 ; j=0,1, \ldots, m-1(N(\varepsilon)$ можно считать не зависяшим от $r, j$ и $x \in[a, b]$, где $[a, b] \subset(0,+\infty))$. Из соотношений $(26),(27)$ следует, что при $n \geqslant N(\varepsilon)$

$$
\begin{aligned}
P_{n}(\alpha, \beta) \leqslant & (1+\varepsilon) \mathbf{P}\left\{\hat{\varphi} \in B_{1}\right\} \mathbf{P}\left\{\check{\varphi} \in B_{2}\right\} \widehat{c} \check{c} \sum_{j=0}^{m-1} \sum_{l=0}^{l-1} \frac{t_{r+1}-t_{r}+1 / n}{\sqrt{t_{r}\left(1-t_{r+1}\right)}} \\
& \times \mathbf{P}\left\{W^{+}(1) \in\left(\frac{y_{j}}{\sqrt{t_{r+1}}}, \frac{y_{j+1}}{\sqrt{t_{r}}}\right], \sup _{0 \leqslant t \leqslant 1}\left[W^{+}(t)-W^{+}(1)\right] \leqslant \frac{x}{\sqrt{t_{r}}}\right\} \\
& \times \mathbf{P}\left\{\sup _{0 \leqslant t \leqslant 1} W^{+}(t) \geqslant \frac{y_{j+1}+x}{\sqrt{1-t_{r+1}}}\right\} .
\end{aligned}
$$

Откуда, переходя к пределу сначала при $n \rightarrow \infty$, а затем при $\max _{0 \leqslant r \leqslant l-1}\left(t_{r+1}-t_{r}\right) \rightarrow 0$, получаем, что

$$
\begin{aligned}
\varlimsup_{n \rightarrow \infty} P_{n}(\alpha, \beta) \leqslant(1+\varepsilon) \mathbf{P}\left\{\hat{\varphi} \in B_{1}\right\} \mathbf{P}\left\{\check{\varphi} \in B_{2}\right\} \hat{c} \check{c} \\
\times \sum_{j=0}^{m-1} \int_{\alpha}^{1-\alpha} \mathbf{P}\left\{W^{+}(1) \in\left(\frac{y_{j}}{\sqrt{t}}, \frac{y_{j+1}}{\sqrt{t}}\right], \sup _{0 \leqslant t \leqslant 1}\left[W^{+}(t)-W^{+}(1)\right] \leqslant \frac{x}{\sqrt{t}}\right\} \\
\times \mathbf{P}\left\{\sup _{0 \leqslant t \leqslant 1} W^{+}(t) \geqslant \frac{y_{j+1}+x}{\sqrt{1-t}}\right\} d t .
\end{aligned}
$$

Наконец, переходя к пределу при $\max _{0 \leqslant j \leqslant m-1}\left(y_{j+1}-y_{j}\right) \rightarrow 0$, находим, что

$$
\varlimsup_{n \rightarrow \infty} P_{n}(\alpha, \beta) \leqslant(1+\varepsilon) \mathbf{P}\left\{\hat{\varphi} \in B_{1}\right\} \mathbf{P}\left\{\check{\varphi} \in B_{2}\right\} \hat{c} \check{c} I(\alpha, \beta),
$$

где

$$
\begin{gathered}
I(\alpha, \beta)=\int_{\alpha}^{1-\alpha} d t \int_{\beta}^{1 / \beta} \mathbf{P}\left\{\sup _{0 \leqslant t \leqslant 1} W^{+}(t) \geqslant \frac{y+x}{\sqrt{1-t}}\right\} d_{\mathbf{y}} \mathbf{P}\left\{W^{+}(1) \leqslant \frac{y}{\sqrt{t}},\right. \\
\left.\sup _{0 \leqslant t \leqslant 1}\left[W^{+}(t)-W^{+}(1)\right] \leqslant \frac{x}{\sqrt{t}}\right\} .
\end{gathered}
$$

Аналогично (28) устанавливается, что

$$
\varliminf_{n \rightarrow \infty} P_{n}(\alpha, \beta) \geqslant(1-\varepsilon) \mathbf{P}\left\{\hat{\varphi} \in B_{1}\right\} \mathbf{P}\left\{\check{\varphi} \in B_{2}\right\} \widehat{c} \check{c} I(\alpha, \beta) .
$$

Ввиду произвольности $\varepsilon$ из (28), (29) следует, что

$$
\lim _{n \rightarrow \infty} P_{n}(\alpha, \beta)=\mathbf{P}\left\{\hat{\varphi} \in B_{1}\right\} \mathbf{P}\left\{\check{\varphi} \in B_{2}\right\} \hat{c} \check{c} I(\alpha, \beta) .
$$

Из принципа инвариантности Донскера-Прохорова вытекает, что

$$
\lim _{n \rightarrow \infty} \mathbf{P}\left\{\min _{0 \leqslant i \leqslant n} \frac{S_{i}}{\sigma \sqrt{n}} \geqslant-x\right\}=\mathbf{P}\left\{\inf _{0 \leqslant t \leqslant 1} W(t) \geqslant-x\right\},
$$


причем эта сходимость равномерна по $x$ на любом отрезке. Из соотношений $(25),(30),(31)$ находим, что

$$
\begin{array}{r}
\lim _{n \rightarrow \infty} \mathbf{P}\left\{\widehat{\varphi}_{n} \in B_{1}, \check{\varphi}_{n} \in B_{2}, \frac{T_{M}}{n} \in(\alpha, 1-\alpha], \frac{S_{T_{M}}}{\sigma \sqrt{n}} \in\left(\beta, \frac{1}{\beta}\right] \mid\right. \\
\left.\min _{0 \leqslant i \leqslant n} \frac{S_{i}}{\sigma \sqrt{n}} \geqslant-x\right\}=\frac{\mathbf{P}\left\{\hat{\varphi} \in B_{1}\right\} \mathbf{P}\left\{\check{\varphi} \in B_{2}\right\} \hat{c} \check{c} I(\alpha, \beta)}{\mathbf{P}\left\{\inf _{0 \leqslant t \leqslant 1} W(t) \geqslant-x\right\}} .
\end{array}
$$

Откуда, переходя к пределу при $\alpha \rightarrow 0, \beta \rightarrow 0$, получаем, что

$$
\begin{gathered}
\lim _{n \rightarrow \infty} \mathbf{P}\left\{\widehat{\varphi}_{n} \in B_{1}, \check{\varphi}_{n} \in B_{2} \mid \min _{0 \leqslant i \leqslant n} \frac{S_{i}}{\sigma \sqrt{n}} \geqslant-x\right\} \\
=\frac{\mathbf{P}\left\{\widehat{\varphi} \in B_{1}\right\} \mathbf{P}\left\{\check{\varphi} \in B_{2}\right\} \widehat{c} \check{c} I(0+, 0+)}{\mathbf{P}\left\{\inf _{0 \leqslant t \leqslant 1} W(t) \geqslant-x\right\}} .
\end{gathered}
$$

Точно так же показывается, что

$$
\begin{aligned}
1 & =\lim _{n \rightarrow \infty} \mathbf{P}\left\{\frac{T_{M}}{n} \in[0,1], \frac{S_{T_{M}}}{\sigma \sqrt{n}} \in[0,+\infty) \mid \min _{0 \leqslant i \leqslant n} \frac{S_{i}}{\sigma \sqrt{n}} \geqslant-x\right\} \\
& =\frac{\widehat{c} \check{c} I(0+, 0+)}{\mathbf{P}\left\{\inf _{0 \leqslant t \leqslant 1} W(t) \geqslant-x\right\}} .
\end{aligned}
$$

Из соотношений (32), (33) следуют соотношение (24) и утверждение леммы 6 .

Лемма 7. Если $\mathbf{E} X_{1}=0,0<\mathbf{E} X_{1}^{2}<+\infty$, то при любом $\delta \in[0,1)$ $u n \rightarrow \infty$

$$
\left\{\varphi_{n}(\delta) \mid \tau_{1}>n\right\} \stackrel{\mathscr{D}}{\longrightarrow} \varphi .
$$

Д о к а з а т е л в с т в о. Для произвольного $\varphi$-непрерывного борелевского множества $B \subset \mathbf{R}$ и произвольного $\delta \in(0,1)$ запишем представление:

$$
\begin{aligned}
& \mathbf{P}\left\{\varphi_{n}(\delta) \in B \mid \tau_{1}>n\right\}=\frac{\mathbf{P}\left\{\tau_{1}>[n \delta]\right\}}{\mathbf{P}\left\{\tau_{1}>n\right\}} \\
& \quad \times \int_{0+}^{+\infty} \mathbf{P}\left\{\frac{\sum_{i=0}^{n-[n \delta]} e^{S_{i}^{\prime}}}{\max _{0 \leqslant i \leqslant n-[n \delta]} e^{S_{i}^{\prime}}} \in B \mid \min _{0 \leqslant i \leqslant n-[n \delta]} \frac{S_{i}^{\prime}}{\sigma \sqrt{n}} \geqslant-x\right\} \\
& \quad \times \mathbf{P}\left\{\min _{0 \leqslant i \leqslant n-[n \delta]} \frac{S_{i}}{\sigma \sqrt{n}} \geqslant-x\right\} d_{x} \mathbf{P}\left\{\frac{S_{[n \delta]}}{\sigma \sqrt{n}} \leqslant x \mid \tau_{1}>[n \delta]\right\},
\end{aligned}
$$

где $S_{i}^{\prime}=S_{[n \delta]+i}-S_{[n \delta]}, i=0,1,2, \ldots$ Случайная последовательность $\left\{S_{i}^{\prime}\right\}$ есть точная вероятностная копия блуждания $\left\{S_{i}\right\}$. По принципу инвариантности Донскера-Прохорова при любом $x \in \mathbf{R}$

$$
\lim _{n \rightarrow \infty} \mathbf{P}\left\{\min _{0 \leqslant i \leqslant n-[n \delta]} \frac{S_{i}^{\prime}}{\sigma \sqrt{n}} \geqslant-x\right\}=\mathbf{P}\left\{\inf _{0 \leqslant t \leqslant 1} W(t) \geqslant \frac{-x}{\sqrt{1-\delta}}\right\},
$$


причем сходимость равномерна на любом отрезке. В силу условного принципа инвариантности Иглхарта при любом $x \in \mathbf{R}$

$$
\lim _{n \rightarrow \infty} \mathbf{P}\left\{\frac{S_{[n \delta]}}{\sigma \sqrt{n}} \leqslant x \mid \tau_{1}>[n \delta]\right\}=\mathbf{P}\left\{W^{+}(1) \leqslant \frac{x}{\sqrt{\delta}}\right\} .
$$

Ввиду соотношения (4)

$$
\lim _{n \rightarrow \infty} \frac{\mathbf{P}\left\{\tau_{1}>[n \delta]\right\}}{\mathbf{P}\left\{\tau_{1}>n\right\}}=\frac{1}{\sqrt{\delta}}
$$

В силу леммы 6 при любом $x \in(0,+\infty)$

$$
\lim _{n \rightarrow \infty} \mathbf{P}\left\{\frac{\sum_{i=0}^{n-[n \delta]} e^{S_{i}^{\prime}}}{\max _{0 \leqslant i \leqslant n-[n \delta]} e^{S_{i}^{\prime}}} \in B \mid \min _{0 \leqslant i \leqslant n-[n \delta]} \frac{S_{i}^{\prime}}{\sigma \sqrt{n}} \geqslant-x\right\}=\mathbf{P}\{\varphi \in B\},
$$

причем эта сходимость равномерна по $x$ на любом отрезке $[a, b] \subset$ $(0,+\infty)$. Из соотношений $(35)-(39)$ следует, что

$$
\begin{aligned}
\lim _{n \rightarrow \infty} \mathbf{P}\left\{\varphi_{n}(\delta) \in B \mid \tau_{1}>n\right\} & =\mathbf{P}\{\varphi \in B\} \delta^{-1 / 2} \\
\times & \int_{0}^{+\infty} \mathbf{P}\left\{\inf _{t \in[0,1]} W(t) \geqslant-\frac{x}{\sqrt{1-\delta}}\right\} d_{x} \mathbf{P}\left\{W^{+}(1) \leqslant \frac{x}{\sqrt{\delta}}\right\} .
\end{aligned}
$$

Точно так же можно доказать, что при любом $\delta \in(0,1)$

$$
\begin{aligned}
1 & =\lim _{n \rightarrow \infty} \mathbf{P}\left\{\tau_{1}>n \mid \tau_{1}>n\right\} \\
& =\frac{1}{\sqrt{\delta}} \int_{0}^{+\infty} \mathbf{P}\left\{\inf _{t \in[0,1]} W(t) \geqslant \frac{-x}{\sqrt{1-\delta}}\right\} d_{x} \mathbf{P}\left\{W^{+}(1) \leqslant \frac{x}{\sqrt{\delta}}\right\} .
\end{aligned}
$$

Из соотношений $(40),(41)$ видно, что при любом $\delta \in(0,1)$

$$
\lim _{n \rightarrow \infty} \mathbf{P}\left\{\varphi_{n}(\delta) \in B \mid \tau_{1}>n\right\}=\mathbf{P}\{\varphi \in B\} .
$$

Осталось доказать (42) при $\delta=0$. Аналогично соотношению (17) можно показать, что при фиксированном $\varepsilon \in(0,+\infty)$

$$
\varlimsup_{\delta \rightarrow 0} \varlimsup_{n \rightarrow \infty} \mathbf{P}\left\{\frac{\sum_{i=0}^{[\delta n]-1} e^{S_{i}}}{\sum_{i=[\delta n]}^{n} e^{S_{i}}}>\varepsilon \mid \tau_{1}>n\right\}=0 .
$$

Аналогично соотношению (22) показывается, что

$$
\lim _{\delta \rightarrow 0} \lim _{n \rightarrow \infty} \mathbf{P}\left\{\max _{0 \leqslant i \leqslant[\delta n]-1} e^{S_{i}}<\max _{[\delta n] \leqslant i \leqslant n} e^{S_{i}} \mid \tau_{1}>n\right\}=1 .
$$

Из (42)-(44) следует, что соотношение (42) справедливо и при $\delta=0$. Лемма 7 доказана.

Лемма 8. Если $\mathbf{E} X_{1}=0,0<\mathbf{E} X_{1}^{2}<+\infty$, то при любых $\delta \in[0,1)$, $j \in \mathbf{N}_{0} u n \rightarrow \infty$

$$
\left\{\varphi_{n}(\delta) \mid \tau_{j} \leqslant n<\tau_{j+1}\right\} \stackrel{\mathscr{D}}{\longrightarrow} \varphi .
$$


Д ок а 3 а т ел ь с т в. При $j=0$ соотношение (45) составляет содержание леммы 7. Рассмотрим $j \in \mathbf{N}$ и $\delta \in(0,1)$. В работе [4] показано, что в условиях доказываемой леммы при любом фиксированном $\lambda \in(0,1)$

$$
\lim _{n \rightarrow \infty} \frac{\mathbf{P}\left\{\tau_{j} \leqslant \lambda n, n<\tau_{j+1}\right\}}{\mathbf{P}\left\{\tau_{j} \leqslant n<\tau_{j+1}\right\}}=1
$$

Поэтому с учетом соотношения (4) достаточно показать, что для произвольного $\varphi$-непрерывного борелевского множества $B \subset \mathbf{R}$ и $\lambda \in(0, \delta)$

$$
\lim _{n \rightarrow \infty} \mathbf{P}\left\{\varphi_{n}(\delta) \in B \mid \tau_{j} \leqslant \lambda n, n<\tau_{j+1}\right\}=\mathbf{P}\{\varphi \in B\} .
$$

Справедливо представление:

$$
\begin{aligned}
& \mathbf{P}\left\{\varphi_{n}(\delta) \in B \mid \tau_{j} \leqslant \lambda n, n<\tau_{j+1}\right\}=\left(\mathbf{P}\left\{\tau_{j} \leqslant \lambda n, n<\tau_{j+1}\right\}\right)^{-1} \\
& \times \sum_{k=1}^{[\lambda n]} \mathbf{P}\left\{\frac{\sum_{i=[\delta n]-k}^{n-k} e^{S_{i}^{\prime}}}{\max _{[\delta n]-k \leqslant i \leqslant n-k} e^{S_{i}^{\prime}}} \in B, \tau_{j}=k, \tau_{1}^{\prime}>n-k\right\} \\
&= \sum_{k=1}^{[\lambda n]} \frac{\mathbf{P}\left\{\tau_{j}=k\right\} \mathbf{P}\left\{\tau_{1}^{\prime}>n-k\right\}}{\mathbf{P}\left\{\tau_{j} \leqslant \lambda n, n<\tau_{j+1}\right\}} \\
& \quad \times \mathbf{P}\left\{\frac{\sum_{i=[\delta n]-k}^{n-k} e^{S_{i}^{\prime}}}{\max _{[\delta n]-k \leqslant i \leqslant n-k} e^{S_{i}^{\prime}}} \in B \mid \tau_{1}^{\prime}>n-k\right\} \\
&=\sum_{k=1}^{[\lambda n]} \frac{\mathbf{P}\left\{\tau_{j}=k\right\} \mathbf{P}\left\{\tau_{1}>n-k\right\}}{\mathbf{P}\left\{\tau_{j} \leqslant \lambda n, n<\tau_{j+1}\right\}} \\
& \times \mathbf{P}\left\{\frac{\sum_{i=[\delta n]-k}^{n-k} e^{S_{i}}}{\max _{[\delta n]-k \leqslant i \leqslant n-k} e^{S_{i}}} \in B \mid \tau_{1}>n-k\right\}
\end{aligned}
$$

где в случае $\left\{\tau_{j}=k\right\} \quad S_{i}^{\prime}=S_{k+i}-S_{k}, i=0,1, \ldots ; \tau_{1}^{\prime}=\tau_{j+1}-\tau_{j}$. Мы воспользовались тем, что случайная последовательность $\left\{S_{i}^{\prime}\right\}$ является точной вероятностной копией блуждания $\left\{S_{i}\right\} ; \tau_{1}^{\prime}$ оказывается первым строгим нижним лестничным моментом блуждания $\left\{S_{i}^{\prime}\right\}$; блуждания $\left\{S_{i}^{\prime}\right\}$ и $\left\{S_{i}\right\}$ являются независимыми. При произвольном фиксированном $k \in \mathbf{N}$

$$
\lim _{n \rightarrow \infty} \mathbf{P}\left\{\frac{\sum_{i=[\delta n]-k}^{n-k} e^{S_{i}}}{\max _{[\delta n]-k \leqslant i \leqslant n-k} e^{S_{i}}} \in B \mid \tau_{1}>n-k\right\}=\mathbf{P}\{\varphi \in B\} .
$$

Действительно, соотношение (49) равносильно утверждению, что

$$
\lim _{n \rightarrow \infty} \mathbf{P}\left\{\frac{\sum_{i=[\delta(n+k)]-k}^{n} e^{S_{i}}}{\max _{[\delta(n+k)]-k \leqslant i \leqslant n} e^{S_{i}}} \in B \mid \tau_{1}>n\right\}=\mathbf{P}\{\varphi \in B\},
$$


которое вытекает из леммы 7 и следуюших соотношений: при $\varepsilon \in$ $(0,+\infty)$

$$
\begin{aligned}
& \lim _{n \rightarrow \infty} \mathbf{P}\left\{\frac{\sum_{i=[\delta(n+k)]-k}^{[\delta n]-1} e^{S_{i}}}{\sum_{i=[\delta n]}^{n} e^{S_{i}}}>\varepsilon \mid \tau_{1}>n\right\}=0, \\
& \lim _{n \rightarrow \infty} \mathbf{P}\left\{\max _{[\delta(n+k)]-k \leqslant i \leqslant[\delta n]-1} e^{S_{i}}<\max _{[\delta n] \leqslant i \leqslant n} e^{S_{i}} \mid \tau_{1}>n\right\}=1,
\end{aligned}
$$

доказываюшихся аналогично соотношениям (17) и (22). Из соотношений (4), (46) и (49) следует, что предел при $n \rightarrow \infty$ отдельного слагаемого в правой части соотношения (48) равен $\mathbf{P}\left\{\tau_{j}=k\right\} \mathbf{P}\{\varphi \in B\}$. Более того, при достаточно больших $n$ и всех $k=1,2, \ldots,[\lambda n]$

$$
\begin{aligned}
& \frac{\mathbf{P}\left\{\tau_{j}=k\right\} \mathbf{P}\left\{\tau_{1}>n-k\right\}}{\mathbf{P}\left\{\tau_{j} \leqslant \lambda n, n<\tau_{j+1}\right\}} \mathbf{P}\left\{\frac{\sum_{i=[\delta n]-k}^{n-k} e^{S_{i}}}{\max _{[\delta n]-k \leqslant i \leqslant n-k} e^{S_{i}}} \in B \mid \tau_{1}>n-k\right\} \\
& \quad \leqslant \frac{\mathbf{P}\left\{\tau_{j}=k\right\}(1+\varepsilon)}{\sqrt{1-\lambda}} .
\end{aligned}
$$

Следовательно, предел правой части соотношения (48) равен $\mathbf{P}\{\varphi \in B\}$. Итак, соотношение (47) и, значит, (45) при $\delta \in(0,1)$ доказаны. Отсюда получаем соотношение (45) при $\delta=0$ (см. окончание доказательства леммы 7, при этом нужно использовать обобщение условного принципа инвариантности Иглхарта с заменой условия $\left\{\tau_{1}>n\right\}$ на условие $\left\{\tau_{j} \leqslant\right.$ $\left.n<\tau_{j+1}\right\}$ (см. [2])). Лемма 8 доказана.

Пусть $\left\{\bar{Y}_{i}, i \in \mathbf{N}\right\}$ - последовательность случайных векторов одинаковой размерности такая, что случайные векторы $\left(X_{1}, \bar{Y}_{1}\right),\left(X_{2}, \bar{Y}_{2}\right), \ldots$ независимы и одинаково распределены. В работе [1] установлен следующий результат.

Лемма 9. Если $\mathbf{E} X_{1}=0 u 0<\mathbf{E} X_{1}^{2}<+\infty$, то при любом $j \in \mathbf{N}_{0}$ $u n \rightarrow \infty$

$$
\left\{\left(S_{i}, \bar{Y}_{i}\right), i \in \mathbf{N} \mid \tau_{j} \leqslant n<\tau_{j+1}\right\} \stackrel{\mathscr{D}}{\longrightarrow}\left\{\left(S_{i}^{(j)}, \bar{Y}_{i}^{(j)}\right), i \in \mathbf{N}\right\}
$$

где справа стоит некоторая последовательность случайных векmоров.

Лемма 10. Если $\mathbf{E} X_{1}=0$ u $0<\mathbf{E} X_{1}^{2}<+\infty$, то при любьи $j \in \mathbf{N}_{0}, \delta \in[0,1)$ u $n \rightarrow \infty$ случайная последовательность $\left\{\left(S_{i}, \bar{Y}_{i}\right)\right.$, $i \in \mathbf{N}\}$ и случайная величина $\varphi_{n}(\delta)$, рассматриваемые при условии $\left\{\tau_{j} \leqslant n<\tau_{j+1}\right\}$, асимптотически независимы (в смысле конечномерных распределений).

Доказ а тель с т о. Пусть размерность вектора $\bar{Y}_{1}$ равна $l$. Рассмотрим сначала случай, когда $j=0$. Для $\delta \in(0,1)$, произвольного $m \in \mathbf{N}$ и произвольных борелевских множеств $B \subset \mathbf{R}, B_{i} \in \mathbf{R}^{l+1}$, 
$i=1, \ldots, m$, запишем при достаточно больших $n$ представление:

$$
\begin{aligned}
& \mathbf{P}\left\{\left(S_{i}, \bar{Y}_{i}\right) \in B_{i}, i=1, \ldots, m ; \varphi_{n}(\delta) \in B \mid \tau_{1}>n\right\} \\
& =\frac{\mathbf{P}\left\{\tau_{1}>[n \delta]\right\}}{\mathbf{P}\left\{\tau_{1}>n\right\}} \int_{0}^{+\infty} \mathbf{P}\left\{\frac{\sum_{i=0}^{n-[n \delta]} e^{S_{i}^{\prime}}}{\max _{0 \leqslant i \leqslant n-[n \delta]} e^{S_{i}^{\prime}}} \in B \mid \min _{0 \leqslant i \leqslant n-[n \delta]} \frac{S_{i}^{\prime}}{\sigma \sqrt{n}} \geqslant-x\right\} \\
& \quad \times \mathbf{P}\left\{\min _{0 \leqslant i \leqslant n-[n \delta]} \frac{S_{i}^{\prime}}{\sigma \sqrt{n}} \geqslant-x\right\} d_{x} \mathbf{P}\left\{\begin{array}{c}
\left(S_{i}, \bar{Y}_{i}\right) \in B_{i}, i=1, \ldots, m ; \\
\left.\frac{S_{[n \delta]}}{\sigma \sqrt{n}} \leqslant x \mid \tau_{1}>[n \delta]\right\},
\end{array}\right.
\end{aligned}
$$

где $S_{i}^{\prime}=S_{[n \delta]+i}-S_{[n \delta]}, i=0,1, \ldots$ Из этого представления выводится утверждение доказываемой леммы при $j=0$ точно так же, как утверждение леммы 7 выводится из представления (35), при этом надо использовать доказанную в работе [10] асимптотическую независимость при $n \rightarrow \infty$ (в смысле конечномерных распределений) случайной последовательности $\left\{\left(S_{i}, \bar{Y}_{i}\right), i \in \mathbf{N}\right\}$ и случайного процесса $\left\{S_{[n t]} /(\sigma \sqrt{n})\right.$, $t \in[0,+\infty)\}$, рассматриваемых при условии $\left\{\tau_{1}>n\right\}$.

Пусть теперь $j \in \mathbf{N}$. При $\delta \in(0,1), \lambda \in(0, \delta)$ и достаточно больших $n$ запишем представление:

$$
\begin{gathered}
\mathbf{P}\left\{\left(S_{i}, \bar{Y}_{i}\right) \in B_{i}, i=1, \ldots, m ; \varphi_{n}(\delta) \in B \mid \tau_{j} \leqslant \lambda n, n<\tau_{j+1}\right\} \\
=\sum_{k=1}^{[\lambda n]} \mathbf{P}\left\{\left(S_{r}, \bar{Y}_{r}\right) \in B_{r}, r=1, \ldots, k \wedge m ;\left(S_{k}+S_{i}^{\prime}, Y_{i}^{\prime}\right) \in B_{k+i},\right. \\
i=1, \ldots, m-k \wedge m ; \frac{\sum_{i=[\delta n]-k}^{n-k} e^{S_{i}^{\prime}}}{\max _{[\delta n]-k \leqslant i \leqslant n-k} e^{S_{i}^{\prime}}} \in B ; \\
\left.\tau_{j}=k \mid \tau_{1}^{\prime}>n-k\right\} \frac{\mathbf{P}\left\{\tau_{1}^{\prime}>n-k\right\}}{\mathbf{P}\left\{\tau_{j} \leqslant \lambda n, n<\tau_{j+1}\right\}},
\end{gathered}
$$

где в случае $\left\{\tau_{j}=k\right\} \quad S_{i}^{\prime}=S_{k+i}-S_{k}, Y_{i}^{\prime}=Y_{k+i}, i=0,1, \ldots ; \tau_{1}^{\prime}=$ $\tau_{j+1}-\tau_{j}$. Из этого представления, учитывая независимость блужданий $\left\{S_{i}\right\}$ и $\left\{S_{i}^{\prime}\right\}$, а также уже полученное при $j=0$ утверждение рассматриваемой леммы, можно получить это утверждение при $j \in \mathbf{N}$ точно так же, как лемма 8 получается из представления (48). Лемма 10 доказана.

Лемма 11. В условиях теоремы 3 при $n \rightarrow \infty$

$$
\left\{\varphi_{n}, \frac{\xi_{n}}{m_{n}} \mid T>n\right\} \stackrel{\mathscr{D}}{\longrightarrow}(\varphi, \mu),
$$

где случайные величины $\varphi$ и (те же, что и в соотношениях (6) $и$ (1) соответственно) предполагаются независимыми.

Д ок аз а тель с т в. Пусть $B-\varphi$-непрерывное борелевское множество на числовой прямой, $x \in(0,+\infty)$. Для нахождения предела 
вероятности $\mathbf{P}\left\{\varphi_{n} \in B, \xi_{n} / m_{n}>x \mid T>n\right\}$ при $n \rightarrow \infty$ в силу леммы 1 достаточно при каждом $j \in \mathbf{N}_{0}$ найти предел вероятности $\mathbf{P}\left\{\varphi_{n} \in B\right.$, $\left.\xi_{n} / m_{n}>x, T>n \mid \tau_{j} \leqslant n<\tau_{j+1}\right\}$ или, что то же самое, $\mathbf{P}\left\{\varphi_{n} \in B\right.$, $\left.\xi_{n} / m_{n}>x \mid \tau_{j} \leqslant n<\tau_{j+1}\right\}$. В работе [1] показано, что в условиях теоремы 1 при любых $j \in \mathbf{N}_{0}$ и $\varepsilon \in(0,+\infty)$

$$
\lim _{k \rightarrow \infty} \varlimsup_{n \rightarrow \infty} \mathbf{P}\left\{\left|\frac{\xi_{n}}{m_{n}}-\frac{\xi_{k}}{m_{k}}\right| \geqslant \varepsilon \mid \tau_{j} \leqslant n<\tau_{j+1}\right\}=0 .
$$

Из этого соотношения вытекает, что достаточно найти

$$
\lim _{k \rightarrow \infty} \lim _{n \rightarrow \infty} \mathbf{P}\left\{\varphi_{n} \in B, \frac{\xi_{k}}{m_{k}}>x \mid \tau_{j} \leqslant n<\tau_{j+1}\right\} .
$$

В работе [1] показано, что в условиях теоремы 1 при любом $j \in \mathbf{N}_{0}$ и $n \rightarrow \infty$

$$
\left\{\xi_{k}, k \in \mathbf{N}_{0} \mid \tau_{j} \leqslant n<\tau_{j+1}\right\} \stackrel{\mathscr{D}}{\longrightarrow}\left\{\xi_{k}^{(j)}, k \in \mathbf{N}\right\},
$$

где $\left\{\xi_{k}^{(j)}\right\}$ является ветвящимся процессом в некоторой случайной среде $\left\{\pi_{k}^{(j)}\right\}$. Ввиду этого для нахождения предела (51) достаточно найти при достаточно больших $L \in \mathbf{R}$

$$
\lim _{k \rightarrow \infty} \lim _{n \rightarrow \infty} \mathbf{P}\left\{\varphi_{n} \in B, \frac{\xi_{k}}{m_{k}}>x, \max _{0 \leqslant i \leqslant k} \xi_{i} \leqslant L \mid \tau_{j} \leqslant n<\tau_{j+1}\right\} .
$$

Очевидно, что

$$
\mathbf{P}_{\pi}\left\{\frac{\xi_{k}}{m_{k}}>x, \max _{0 \leqslant i \leqslant k} \xi_{i} \leqslant L\right\}=f_{k}\left(S_{1}, \bar{Y}_{1}, \ldots, S_{k}, \bar{Y}_{k}\right),
$$

где $f_{k}-$ некоторая измеримая ограниченная функция, $\bar{Y}_{i}=\left(\pi_{i-1}(0)\right.$, $\left.\pi_{i-1}(1), \ldots, \pi_{i-1}([L])\right), i=1, \ldots, k$. Поэтому

$$
\begin{aligned}
& \mathbf{P}\left\{\varphi_{n} \in B, \frac{\xi_{k}}{m_{k}}>x, \max _{0 \leqslant i \leqslant k} \xi_{i} \leqslant L \mid \tau_{j} \leqslant n<\tau_{j+1}\right\} \\
& =\mathbf{E}\left(\chi_{\left\{\varphi_{n} \in B\right\}} f_{k}\left(S_{1}, \bar{Y}_{1}, \ldots, S_{k}, \bar{Y}_{k}\right) \mid \tau_{j} \leqslant n<\tau_{j+1}\right),
\end{aligned}
$$

где $\chi_{A}$ - индикатор множества $A$. Откуда в силу лемм 8-10 при почти всех $x, L$

$$
\begin{aligned}
& \lim _{n \rightarrow \infty} \mathbf{P}\left\{\varphi_{n} \in B, \frac{\xi_{k}}{m_{k}}>x, \max _{0 \leqslant i \leqslant k} \xi_{i} \leqslant L \mid \tau_{j} \leqslant n<\tau_{j+1}\right\} \\
& =\mathbf{P}\{\varphi \in B\} \mathbf{E} f_{k}\left(S_{1}^{(j)}, \bar{Y}_{1}^{(j)}, \ldots, S_{k}^{(j)}, \bar{Y}_{k}^{(j)}\right) .
\end{aligned}
$$

Из результатов работы [1] следует, что в условиях теоремы 1

$$
f_{k}\left(S_{1}^{(j)}, \bar{Y}_{1}^{(j)}, \ldots, S_{k}^{(j)}, \bar{Y}_{k}^{(j)}\right)=\mathbf{P}_{\pi^{(j)}}\left\{\frac{\xi_{k}^{(j)}}{m_{k}^{(j)}}>x, \max _{0 \leqslant i \leqslant k} \xi_{i}^{(j)} \leqslant L\right\},
$$


где $m_{k}^{(j)}=\mathbf{E}_{\pi^{(j)}} \xi_{k}^{(j)}=\exp S_{k}^{(j)}$. Итак, доказано, что если $x-$ точка непрерывности функции распределения случайной величины $\xi_{k}^{(j)} / m_{k}^{(j)}$, a $L$ - точка непрерывности функции распределения случайной величины $\max _{0 \leqslant i \leqslant k} \xi_{i}^{(j)}$, то

$$
\begin{gathered}
\lim _{n \rightarrow \infty} \mathbf{P}\left\{\varphi_{n} \in B, \frac{\xi_{k}}{m_{k}}>x, \max _{0 \leqslant i \leqslant k} \xi_{i} \leqslant L \mid \tau_{j} \leqslant n<\tau_{j+1}\right\} \\
=\mathbf{P}\{\varphi \in B\} \mathbf{P}\left\{\frac{\xi_{k}^{(j)}}{m_{k}^{(j)}}>x, \max _{0 \leqslant i \leqslant n} \xi_{i}^{(j)} \leqslant L\right\} .
\end{gathered}
$$

Ввиду соотношения (52) отсюда вытекает, что если $x$ - точка непрерывности функции распределения случайной величины $\xi_{k}^{(j)} / m_{k}^{(j)}$, то

$$
\lim _{n \rightarrow \infty} \mathbf{P}\left\{\varphi_{n} \in B, \frac{\xi_{k}}{m_{k}}>x \mid \tau_{j} \leqslant n<\tau_{j+1}\right\}=\mathbf{P}\{\varphi \in B\} \mathbf{P}\left\{\frac{\xi_{k}^{(j)}}{m_{k}^{(j)}}>x\right\} .
$$

В работе [1] показано, что в условиях теоремы 1 при $k \rightarrow \infty$

$$
\frac{\xi_{k}^{(j)}}{m_{k}^{(j)}} \stackrel{\mathscr{D}}{\longrightarrow} \mu^{(j)}
$$

где $\mu^{(j)}$ - некоторая неотрицательная случайная величина. Из соотношений $(50),(53)$ и (54) получаем, что если $x$ - точка непрерывности функции распределения случайной величины $\mu^{(j)}$, то

$$
\lim _{n \rightarrow \infty} \mathbf{P}\left\{\varphi_{n} \in B, \frac{\xi_{n}}{m_{n}}>x \mid \tau_{j} \leqslant n<\tau_{j+1}\right\}=\mathbf{P}\{\varphi \in B\} \mathbf{P}\left\{\mu^{(j)}>x\right\} .
$$

Пусть $D$ - множество точек, являюшихся точками непрерывности сразу всех функций распределения случайных величин $\mu^{(j)}$. Очевидно, множество $D$ всюду плотно на числовой прямой. При $x \in D \cap(0,+\infty)$ в силу леммы 1 из соотношения (55) находим, что

$$
\lim _{n \rightarrow \infty} \mathbf{P}\left\{\varphi_{n} \in B, \frac{\xi_{n}}{m_{n}}>x \mid T>n\right\}=\mathbf{P}\{\varphi \in B\} \frac{c_{1}}{c_{2}} \sum_{j=0}^{\infty} \mathbf{P}\left\{\mu^{(j)}>x\right\} .
$$

В работе [1] показано, что в условиях теоремы 1 при $x \in D \cap(0,+\infty)$ $c_{2}^{-1} c_{1} \sum_{j=0}^{\infty} \mathbf{P}\left\{\mu^{(j)}>x\right\}=\mathbf{P}\{\mu>x\}$. Из двух последних соотношений получаем, что в условиях теоремы 1 при $x \in D \cap(0,+\infty)$

$$
\lim _{n \rightarrow \infty} \mathbf{P}\left\{\varphi_{n} \in B, \frac{\xi_{n}}{m_{n}}>x \mid T>n\right\}=\mathbf{P}\{\varphi \in B\} \mathbf{P}\{\mu>x\} .
$$

Чтобы доказать это соотношение при $x \in(-\infty, 0]$, впервые воспользуемся условием $\mu>0$ п.н., из которого следует, что

$$
\lim _{x \downarrow 0} \lim _{n \rightarrow \infty} \mathbf{P}\left\{\frac{\xi_{n}}{m_{n}}>x \mid T>n\right\}=1 .
$$


Из соотношений (56), (57) вытекает, что

$$
\begin{aligned}
\lim _{n \rightarrow \infty} \mathbf{P}\left\{\varphi_{n} \in B \mid T>n\right\} & =\lim _{x \downarrow 0} \lim _{n \rightarrow \infty} \mathbf{P}\left\{\varphi_{n} \in B, \frac{\xi_{n}}{m_{n}}>x \mid T>n\right\} \\
& =\mathbf{P}\{\varphi \in B\} .
\end{aligned}
$$

Таким образом, соотношение (56) доказано при $x=0$ и, следовательно, при $x \in(-\infty, 0)$. Лемма 11 доказана.

Д о к а за т е л с с т о т е о ре м ы 3 . В силу лемм 4,5 и 11 при $n \rightarrow \infty$

$$
\left\{\varphi_{n}, \frac{\sum_{i=0}^{n} \xi_{i}}{\sum_{i=0}^{n} m_{i}}, \frac{\max _{0 \leqslant i \leqslant n} \xi_{i}}{\max _{0 \leqslant i \leqslant n} m_{i}} \mid T>n\right\} \stackrel{\mathscr{D}}{\longrightarrow}\{\varphi, \mu, \mu\},
$$

где случайные величины $\varphi$ и $\mu$ независимы и $\mu>0$ п.н. Разделив вторую компоненту на третью и учитывая, что $m_{i}=\exp S_{i}$, получаем, что при $n \rightarrow \infty$

$$
\left\{\varphi_{n}, \frac{\sum_{i=0}^{n} \xi_{i}}{\max _{0 \leqslant i \leqslant n} \xi_{i}} \varphi_{n}^{-1} \mid T>n\right\} \stackrel{\mathscr{D}}{\longrightarrow}\{\varphi, 1\} .
$$

Откуда, перемножая компоненты, приходим к утверждению теоремы 3.

\section{СПИСОК ЛИТЕРАТУРЫ}

1. АФанасьев В. И. Функциональная предельная теорема для критического ветвящегося процесса в случайной среде. - Дискретн. матем., 2001, т. 13, № 4, с. 73-91.

2. Афанасьев $В$. И. Новая предельная теорема для критического ветвяшегося процесса в случайной среде. - Дискретн. матем., 1997, т. 9, № 3, с. 52-67.

3. Geiger J., Kersting G. The survival probability of a critical branching process in random environment. - Теория вероятн. и ее примен., 2000 , т. 45 , в. 3, с. 607-615.

4. Козлов М.В. Об асимптотике вероятности невырождения критических ветвяшихся процессов в случайной среде. - Теория вероятн. и ее примен., 1976, т. 21, B. 4 , c. $813-825$.

5. Ватутин В. A. Редуцированные ветвящиеся процессы в случайной среде: критический случай. - Теория вероятн. и ее примен., 2002, т. 47, в. 1, с. 21-38.

6. Козлов М.В. Условная функциональная предельная теорема для критического ветвящегося процесса в случайной среде. - Докл. РАН, 1995, т. 344, № 1, с. 1215.

7. АФанасьев В. И. О максимуме критического ветвящегося процесса в случайной среде. - Дискретн. матем., 1999, т. 11, № 2, с. 86-102.

8. АФанасьев $В . И$. О моменте достижения фиксированного уровня критическим ветвяшимся процессом в случайной среде. - Дискретн. матем., 1999, т. 11, № 4, c. $33-47$.

9. Афанасьев В.И. О моменте достижения максимума критическим ветвящимся процессом в случайной среде и остановленным случайным блужданием. - Дискретн. матем., 2000, т. 12, № 2, с. 31-50.

10. Афанасьев В. И. Предельная теорема для критического ветвящегося процесса в случайной среде. - Дискретн. матем., 1993, т. 5, № 1, с. 45-58.

11. Hooghiemstra $G$. Conditioned limit theorems for waiting-time processes of the $M / G / 1$ queue. - J. Appl. Probab., 1983, v. 20, № 3, p. 675-688. 